\title{
A decentralized ITS architecture for efficient distribution of traffic task management
}

\author{
Amilcare Francesco Santamaria*, Mauro Tropea*, Peppino Fazio*, \\ Pierfrancesco Raimondo *, Floriano De Rango* and Miroslav Voznak ${ }^{\dagger}$ \\ ${ }^{*}$ University of Calabria, Rende, Italy \\ $\dagger$ VSB - Technical University of Ostrava, Ostrava, Czech Republic
}

\begin{abstract}
In this paper, the attention is focused on the design of a new multi-layered architecture for vehicular environment. System will be able to gather information from vehicular devices. This will allow system to faster respond at emergency situation such as traffic jams or collisions. Distributed entities work at different layers exploiting cloud and fog computing in order to better distribute tasks along the infrastructure. Lower layer is composed of On-Board Units (OBUs) and RoadSide Units (RSUs) that exploits Vehicular Ad-hoc Network (VANET) protocols for inner VANET communications. At the Edge layer we propose fog computing nodes that gather data from RSUs for local processing and after an aggregation step they send data to an Intelligent Transportation System (ITS) management system. The proposed architecture has the main goal to better respond to the network and traffic dynamics by improving performances of the ITS system.
\end{abstract}

Keywords-Vehicular Ad-hoc Network (VANET), Intelligent Transportation System (ITS), Edge computing

\section{INTRODUCTION}

Every year in the world 1.25 million people die for road accidents an in one third of the cases the fault is the excessive speed. Because of the high number of people involved in car accidents the whole scientific and industrial communities are trying to design and develop new solutions able to tackle these problems. Vehicular environment raised in interest because new technologies can help to improve road safety standards. Moreover, these technologies allow us to collect enormous data about the environment and applying modern methodologies it is possible to act in a real-time way on traffic distribution to avoid traffic jams and reduce emissions. This is possible by developing ITS strategies and collecting big data from roads and environmental sensors. The increasing research and development activity in the ITS [1] environment is due to the huge diffusion of transport vehicles and, proportionally, to the number of accidents that happen. The possibility to use VANET standards and the related road infrastructures could help drivers to avoid accidents in the best case or reduce collision severity in the worst case. However, the road safety can be improved by using new communication standards. For example it is possible to reach this goal introducing into the driving system a new set of information about road conditions or enhancing drivers sight along the road [2]. The

ISBN 978-3-903176-03-4 2018 IFIP strength point of a typical VANET system is to allow multihop communication which makes faster messages dissemination along the network. Recent researches is trying to exploit IEEE $802.11 \mathrm{p}$ to allow multi-hop communications [3] and to make an efficient data dissemination [4], exploiting ad-hoc connections that could be created on the IEEE802.11p-based network. However, it is also important to face off traffic and areas congestion because of their side effects on air and life quality. In these last year even more vehicles are traveling through our cities and often road infrastructure are not enough to tackle the increasing of vehicle flows. For these reasons it is important to develop new strategies able manage efficiently our roads. ITS systems may help us to reach this goal but new network solutions needs to be designed. In this work we present a solution for a more feasible ITS system which is able to exploit the benefits of a cloud solution where several data can be processed simultaneously for applying the best solution in terms of traffic distribution. Moreover, we add an edge layer where several local Local-ITS (L-ITS) instances are placed to manage locally a set of road network clusters, in which each RSU is called to manage its area of coverage.

The main contributions of the paper are the following ones:

- A Multi-layered architecture is proposed to manage Locally and Globally Traffic distribution along the interested area.

- Protocol communication rules and Intra-layer interfaces are proposed to allow a feasible and reliable message exchanging between entities.

- Road monitoring and data gathering mechanism are proposed to support local and global traffic optimization.

- Several Simulation campaigns have been conducted to demonstrate the goodness of proposal. A well known simulator framework has been utilized to realize protocol mechanisms for traffic re-distribution and traveling time reduction.

The paper organization is herein summarized : in Section II describes the proposed architecture, with its level and its main functionalities; the protocol communication is discussed in section III. In section IV main tasks distribution along proposed layer is illustrated; Finally, Section V describes the obtained simulation results and conclusions are summarized in Section VI. 


\section{Proposed Architecture}

The proposed architecture is composed of three layers in order to make the system more scalable, efficient. It is able to respond faster to the dynamics of the network. Moreover, system acts to reduce traffic jams because of local management instances called L-ITS. The high level is composed of the cloud instance of global ITS where the human control, global analysis of collected data and distributed data management system take place. ITS cooperates with the local instances called L-ITS, which are instantiated on edge layer. These two layers are responsible of the management of interested area collecting continuously data from VANET layer. L-ITS exploits connections with RSUs to gather information about the monitored area. Globally, these information reach the cloud where the distributed ITS system tries to optimize vehicle flows by applying decision making algorithm. The output of these algorithms are realized in the real world by activating traffic lights and smart road signs where available. In this way, traffic optimization and a better distribution of vehicle loads along the area roads may help to reduce journey time. This strategy is commonly adopted by ITS but sometime it is not able to face in time traffic issues because of their speed. In fact, this dynamic events cannot be avoided and closer area to the origin of the problem are involved faster. To improve the capability of the system we introduced the L-ITS layer which is responsible of managing local area. Since it is placed closer to the environment to be monitored it is able to quickly recognize a possible issue. Therefore, some actions can be taken in a shorter time in order to avoid the spreading of traffic jams along closer areas. Thus, it may also dynamically change the city zone to restricted or unrestricted by allowing or not traffic flows in some areas. In Fig.1, a possible representation of the designed architecture is depicted. Moreover, the L-ITS can interact with RSUs, this allows ITS to communicate with vehicles. Therefore, it is possible to notify information to the drivers interfacing the OBUs. In fact, each vehicle can re-evaluate its own itinerary based on new path computation with updated cost and constraints made by ITS strategies. The messaging system among vehicles and infrastructure is made exploiting VehicleTo-Infrastructure (V2I) links. Message format is based on the use of Wave Short Message Protocol (WSMP) [6], [7], which allows Vehicle-To-Everything (V2X) communications [8]. The Vehicle-To-Vechicle (V2V) communication link is also used by vehicles to disseminate information as happens in common VANET architectures. Instead, regarding the Traffic lights and road signs they are controlled directly by L-ITS exploiting wireless link. In this communication segment we propose to use Machine to Machine (M2M) communication protocol. Regarding the core network, wired connections are used because of the delay constraints.

\section{Protocol Communications}

The overall system is based on the inter and intra communications between layers. We design an edge based architecture for better distribute the computational load allowing

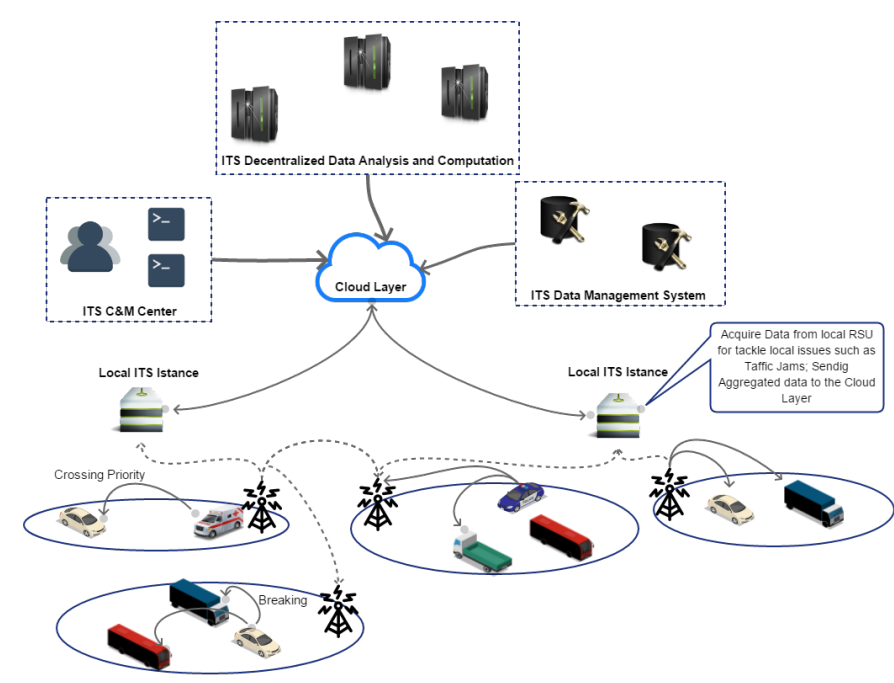

Fig. 1. The proposed multi-layered architecture used for improve road safety and reduce traffic jams.

faster responses. The presented architecture is based on three different layers that perform traffic management tasks. Each layer can communicate with its surrounding layers exploiting dedicated interfaces. Then, a communication protocol uses these interfaces for sending messages between layers. Herein interfaces are summarized:

High ITS-Interface (HITS-I): it defines rules for exchanging data between L-ITS and ITS layers;

ITS-Interface (ITS-I): This interface provides communication between L-ITS;

Low ITS-Interface (LITS-I): it is used to exchange data between L-ITS and RSUs;

VANET Interface (VI): it is used by RSUs to implement $\mathrm{V} 2 \mathrm{~V}$ and V2I interfaces;

\section{A. Vehicle Registration}

In this section the behavior of vehicles is described in detail. When a vehicle begins its journey, it evaluates exploiting the knowledge of the map the Road_ID which it is starting. It sends a Registration request in a broadcast way with a WSMP. Several RSU may receive this message but only the RSU that manages those Road_ID registers the vehicle. It stores vehicle data in a dedicated memory area and generates an Acknowledgment message. Thus, no more register messages will be generated by the vehicle until it changes road. If no acknowledgment is received within a given timeout then another register message is sent. Moreover, this data aggregated data about registrations is collected to evaluate flow rates and road loads.

\section{B. Issues management}

In case of collisions or congestion, we proposed the following protocol rules. In Fig. 2 the collision management and the messages exchange between vehicles is shown. These procedures may help to improve the capability of the system to tackle this event. In particular, the event management is 
composed of several steps. In the reference scenario two vehicles generate a collision, suddenly vehicle generates a beacon message based on WAVE Short Message (WSM) protocol to inform RSU device to spread this event in the area. Moreover, this message is sent towards the L-ITS responsible of the area. It starts to manage the event by updating the status of the lanes involved into the collision. However, sometime local traffic management was not enough to solve traffic issues as shown in Fig. 2. In this case, the ITS takes global decision to reduce congestion in the area of collisions blocking or limit incoming flows from surrounding areas which are managed by different L-ITSs. In order to better distribute message related to a specific event, messages are propagated towards its neighbors L-ITSs by using the ITS-I and towards the cloud instance of ITS by exploiting the HITS-I. L-ITSs provides information about collisions and its decisions such as forbidden access to a given area to the ITS regarding its area of interest. ITS will take under consideration these messages for the next map update process.

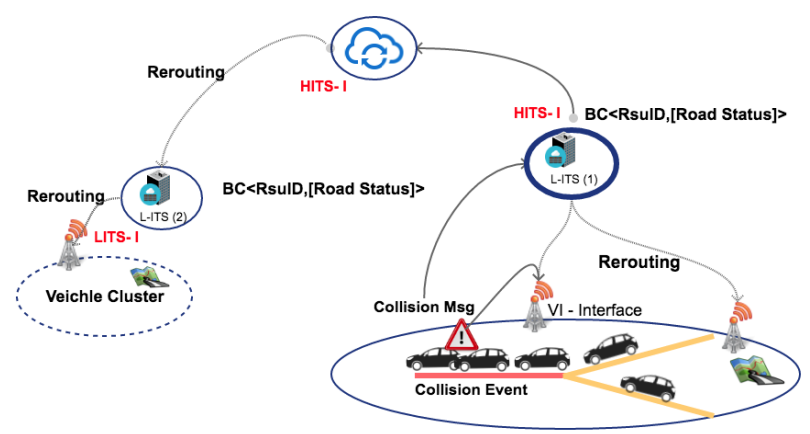

Fig. 2. Collision management by sub-layers. Here vehicles and L-ITS disseminate messages.

\section{DistRIBUTED ITS TASKS}

ITS tasks are distributed among global ITS and L-ITS. In this section, the main idea focuses on the possibility to exploit data collected by vehicles enhancing the capability of ITS to provide traffic solutions as fast as possible. This is achieved through the distribution of tasks along the defined layers. Fog computing [9] may help to reduce system response time analyzing data gathered from environmental sensors, speeding up the decision making processes. In this work, data are provided by vehicles and RSUs. These data are utilized to keep updated roads status.

\section{A. Road Management}

In this sub-section, we introduce how the area of interest is modeled to apply the proposed idea as in [10], [11] in which the following approach has been introduced.

The road network is modeled as a weighted oriented graph, $G=<V, E>$, where $V$ is the set of vertexes, $\|V\|=1,, c$ and $E$ is the set of edges, $\|E\|=1,, m$.

Each edge $e \in E$ represents a lane segment between two vertexes and it has an associated weight $w \in W$ the number of vehicles on the lane, each vertex $v \in V$ represents a cross roads or two lane junction. Fig. 3 illustrates the considered model. To be noted that the graph is undirected, $e_{i, j} \neq e_{j, i}$.

Based on the definition and studies in [12] a density term can be defined as follows:

$$
\begin{aligned}
\left(\pi_{k}\right)_{i, j} & =\frac{\left(w_{k}\right)_{i, j}}{\text { length }\left(e_{i, j}\right)} \\
\Pi_{i, j} & =\sum_{k=1}^{N_{i, j}}\left(\pi_{k}\right)_{i, j}
\end{aligned}
$$

where $\pi_{k_{i, j}}$ is the traffic density of the $k$-th sub-lane of the lane segment $e_{i, j}$ (see Fig. 3 Lane 7-> 8), defined as the ratio of $w_{k_{i, j}}$ (that is the weight of the $k$-th sub-lane of the lane segment $e_{i, j}$ ) and the length $l_{i, j}$ of the lane $e_{i, j}$, while $\Pi_{u, z}$ is the average density for the edge $u, z$ and $N_{i, j}$ is the number of considered lane segments (i.e., in Fig. 3 it is possible to note that a road between node 7 and node 8 is composed of 3 lanes) with $k=1, \ldots, N_{i, j}$. It is necessary to introduce $k$ in order to take into account the possibility to have more lanes with same direction in a same road. In particular, from [13], [14] it can be written that for a motorway the capacity $c_{i, j}$ of the lane $e_{i, j}$ is:

$$
c_{i, j}=C_{i, j} \cdot F H W_{i, j} \cdot F W_{i, j} \cdot F P_{i, j}
$$

where $C_{i, j}$ is the ideal capacity, $F H W_{i, j}$ is related to the probability of having heavy vehicles, $F W_{i, j}$ is a factor related to the width of $e_{i, j}$, and $F P_{i, j}$ is a factor related to the driver population. The criterion for choosing the best path is now illustrated. The algorithm (based on Shortest Path First $(\mathrm{SPF})$ ) is based on reducing the instantaneous road density on all segments and, consequently, the time spent on the road segments. The best path is chosen by:

$$
B E S T \_\operatorname{path}_{k}(D)=\arg \min _{k}\left\{M C A R S\left(\operatorname{path}_{k}(D)\right)\right\}
$$

where $\operatorname{path}_{k}(D)$ is the $k$-th available path to reach the destination $D$. The $M C A R S$ term represents the considered metric. Considering the lane $e_{i, j}$ belonging to the $\operatorname{path}_{k}(D)$, it is defined as:

$$
\begin{array}{r}
M C A R S_{i, j}=\alpha \cdot\left[\frac{w_{i, j} / \text { length }\left(e_{i, j}\right)}{\text { max_} \pi_{\_p a t h}(D)}\right]+(1-\alpha) . \\
{\left[\cdot \frac{\text { length }\left(e_{i, j}\right) / \bar{\nu}_{i, j}}{\text { max_delay_path }(D)}\right]}
\end{array}
$$

where $\alpha$ is a weight term (for giving more emphasis to density or to travel delay), max_ $\pi \_p a t h(D)$ and max_delay_path $(D)$ are the maximum density and delay terms over all paths to destinations and $\bar{\nu}_{i, j}$ is the average speed on lane $e_{i, j}$. Given a path $(D)$ composed by a sequence of segments, the total MCARS[path $(D)]$ is just the sum of the each $M C A R S_{i, j}$ associated to each segment of path $(D)$. 

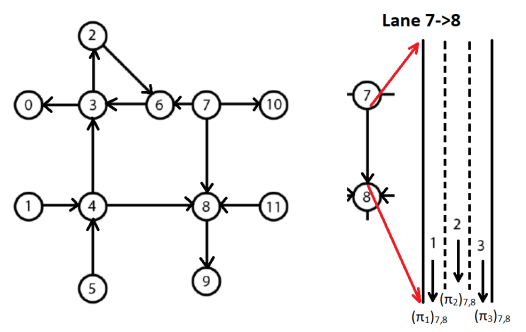

Fig. 3. An example of the way a graph can be associated to a geographical map with a set of roads. To be noted that the graph is unidirected, $e_{i, j} \neq e_{j, i}$.

TABLE I

SIMULATION PARAMETERS

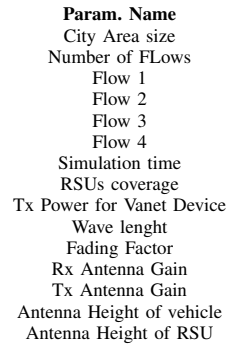

Param. Value
$11 \mathrm{Km} \times 12 \mathrm{Km}$
$1-4$
$[50-300]$
$[50-300]$
$[50-300]$
$[50-300]$
From $6 \mathrm{am}$ to $11 \mathrm{pm}$
$[150-400] \mathrm{mt}$
$20 \mathrm{dBm}$
0.051
2
2
2
$1.50 \mathrm{mt}$
$3.0 \mathrm{mt}$
Description
City map area size managed by ITS
Regulate vehicle density in the monitored area
From $8 \mathrm{am}$ to $12 \mathrm{pm}$ : Vehicle per Hour $(\mathrm{Vph})$
From $8 \mathrm{am}$ to $2 \mathrm{pm}$ : Vehicle per Hour (Vph)
From $8 \mathrm{am}$ to $3 \mathrm{pm}$ : Vehicle per Hour (Vph)
From $12 \mathrm{am}$ to $10 \mathrm{pm}$ : Vehicle per Hour (Vph)
me of the day take into consideration for sims
Coverage radius area for RSU devices
$5.0 \mathrm{GHz}$ of frequency (IEEE $802.11 \mathrm{p})$
fading factor in the vehicular environment
Rx antenna gain
Tx antenna gain
Vehicular antenna height from the ground
Rsu antenna height from the ground

\section{B. Road Monitoring}

Road monitoring is an activity made by L-ITSs. It is made by exploiting data gathering by low layer devices such as RSU and vehicles. In details, it is possible to achieve data in a real-time way about roads status by elaborating messages exchanged in the VANET layer along the VI. In this work the L-ITS gather data from RSUs from WSMP messages such as POSition Updates (POSUPs) and Warnings messages. Since RSUs know how vehicles were moving along roads, it is easy to retrieve number of vehicles per road or lane. It is possible to understand the density of flows as well as road load status by measuring the lane density as shown in Equation (1). Warning messages, instead, can be used to understand if something is happening on a particular lane or road by analyzing the types of warning. Thanks to these data, it will be possible to react to dynamic events by actuating local policies for tackling congestions and collision side effects on traffic.

\section{Performance Evaluation}

In this section, the proposed architecture is evaluated to analyze its behavior in case of raising congestion or collision severity.

\section{A. Simulations Environment}

In order to perform our simulations, we designed a custom simulator using the OMNet++ framework [15] building adhoc modules for the architecture implementation. Protocol messages and interfaces have been implemented in compound modules written in c++ language by extending Veins and Inet module. In particular, Inet framework has been utilized for creating the edge/fog layers and cloud services. These custom

modules permit to realize the L-ITS and ITS modules, with the related communication interfaces. Vehicle environment has been realized by using the Veins module [16]. For creating the mobility scenarios we connect SUMO framework [17] with Veins. Table I summarizes simulation parameters utilized.

\section{B. Proposed Architecture Evaluation}

Our proposal is compared with the common protocol used in VANET environments for signaling warning to others vehicles and another protocol based on cloud services where ITS runs its tasks to reassign traffic load around the covered area.

First of all, it is important to evaluate how the ITS system instantiated in the higher architecture layer impacts on traffic conditions. Regarding ITS implementation and its behavior we implemented a solution already proposed in the [18] where the Ad-hoc On-demand Distance Vector (AODV) protocol is used to disseminate ITS messages in the VANET environment. In Figure 4 (a), the trend of average time that a vehicle spends in the city is depicted. In order to perform this simulation campaign we consider two kinds of collision with a low, medium and high severity degree. Here the average duration of collisions are 10, 15 and 20 minutes respectively. In case of increasing the map update time, the traveling time increases. In this scenario, we evaluate the capability of the system to respond at collision events or traffic jams. This behavior can be observed because system redistributes traffic density too slowly and it involves more areas. In this way, it is clear that ITS solutions only based on centralized services may not be enough to face congestion issues. In Figure 4 (b), it is possible to note that the increasing of update interval increases vehicle density in some area. This issue increases the collision probability. In case of high severity the number of measured collisions are lesser than medium severity because of a lesser average speed.

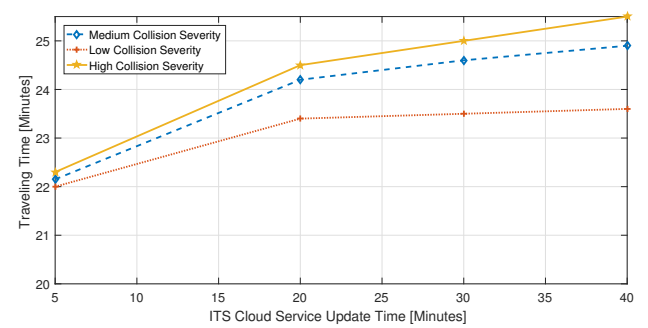

(a)

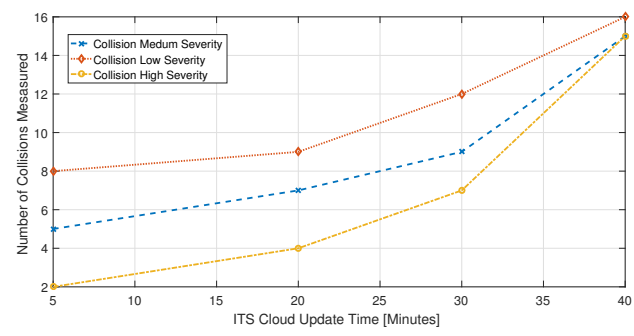

(b)

Fig. 4. (a) Average Traveling Time experienced by vehicle vs. Map Update Time; (b) Number of collision versus Map update Time. 
In this last scenario the ITS centralized approach is compared with the distributed and multi-layered proposal. In Figure 5 (a) the trend of traveling time is reported. It has been demonstrated that, in the case of the distributed approach, it is possible to reduce the queue waiting time by approaching faster to traffic issues, such as collisions or congestions. This allows system to respond faster to these events by rerouting vehicles and avoiding local congestions.

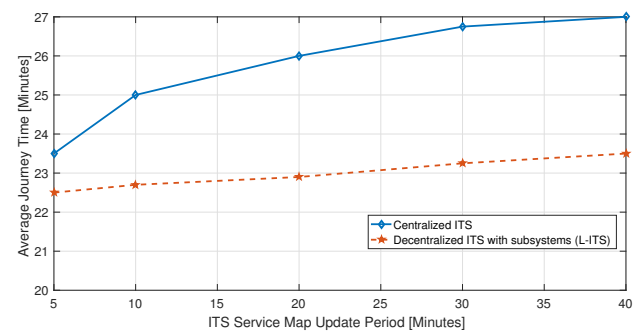

(a)

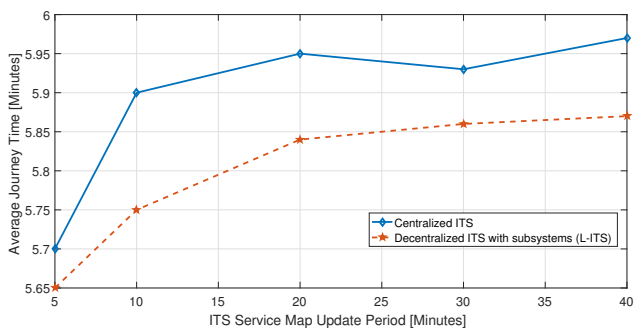

(b)

Fig. 5. (a) Average Traveling time spent by vehicles in the city map area. Here Centralized vs. Decentralized ITS behaviors are depicted; (b) Average Traveled Distance measured by vehicles.

In Figure 5 (b) the average traveled distance per vehicle is depicted for centralized and decentralized ITS solutions. Here it is shown that the decentralized approach allows vehicles to travel a shorter distance than the centralized one. Since centralized ITS starts to react to events only when updated information are received, vehicle rerouting can be performed only after global map update. Moreover, it is demonstrated how the map updated interval influences the goodness of the found solution. With the increasing of the update interval, an increasing of the severity of the traffic jams in the surrounding areas has been observed. The result is the increasing of the traveled distance because of higher density factor per involved lane.

\section{CONCLUSION}

In this work, a new distributed architecture is proposed to improve ITS management for enhancing the performance of traffic solutions. The main idea is to spread information collected by vehicles in the high architecture layers, distributing the elaboration tasks between local and global devices. In particular, the work shows how it is possible to attribute global policies and decision to ITS to perform rerouting policies to balance vehicle density among lanes to mitigate congestion and reduce the experienced traveling time. On the other side the L-ITS can perform local policies to take quick decision for tackling collisions or traffic jams events. Moreover, a protocol has been proposed to allow a more reliable and efficient data dissemination between layers. Finaly simulative campaigns have been carried out to demonstrate the goodness of proposal in terms of number of collision and travelling time.

\section{REFERENCES}

[1] Zhang, J.; Wang, F.Y.; Wang, K.; Lin, W.H.; Xu, X.; Chen, C. DataDriven Intelligent Transportation Systems: A Survey. IEEE Trans. Intell. Transp. Syst. 2011, 12, 1624-1639.

[2] Kurmis, M.; Voznak, M.; Kucinskas, G.; Drungilas, D.; Lukosius, Z.; Jakovlev, S.; Andziulis, A. Development of method for service support management in vehicular communication networks. Adv. Electr. Electron. Eng. 2017, 15, 598-605.

[3] Cumbal, R.; Palacios, H.; Hincapie, R. Optimum deployment of RSU for efficient communications multi-hop from vehicle to infrastructure on VANET. In Proceedings of the 2016 IEEE Colombian Conference on Communications and Computing (COLCOM), Cartagena, Colombia, 2729 April 2016; pp. 1-6.

[4] Kurmis, M.; Andziulis, A.; Dzemydiene, D.; Jakovlev, S.; Voznak, M.; Gricius, G. Cooperative Context Data Acquisition and Dissemination for Situation Identification in Vehicular Communication Networks. Wirel. Pers. Commun. 2015, 85, 49-62.

[5] Sun, Z.; Bebis, G.; Miller, R. On-road vehicle detection using optical sensors: A review. In Proceedings of the 7th International IEEE Conference on Intelligent Transportation Systems (IEEE Cat. No.04TH8749), Washington, WA, USA, 3-6 October 2004; pp. 585-590.

[6] IEEE Standard Committee. IEEE Standard for Wireless Access in Vehicular Environments (WAVE)Networking Services; IEEE Std 1609; IEEE Standard Committee: Piscataway, NJ, USA, 2010; pp. 3-2010.

[7] Li, Y.J. An overview of the DSRC/WAVE technology. In Proceedings of the Conference on Heterogeneous Networking for Quality, Reliability, Security and Robustness, Houston, TX, USA, 17-19 November 2010; Springer: Berlin, Heidelberg, 2010; pp. 544-558.

[8] Schunemann, B.; Massow, K.; Radusch, I. A Novel Approach for Realistic Emulation of Vehicle-2-X Communication Applications. In Proceedings of the Vehicular Technology Conference (VTC Spring 2008), Singapore, 11-14 May 2008; pp. 2709-2713.

[9] Kai, K.; Cong, W.; Tao, L. Fog computing for vehicular Ad-hoc networks: Paradigms, scenarios, and issues. J. China Univ. Posts Telecommun. 2016 , 23, 56-96.

[10] Marshall, S. Line structure representation for road network analysis. $J$. Transp. Land Use 2016, 9, 29-64.

[11] Masucci, A.P.; Stanilov, K.; Batty, M. Exploring the Evolution of Londons Street Network in the Information Space: A Dual Approach. Phys. Rev. E 2014, 89, 012805.

[12] Garavello, M.; Piccoli, B. Traffic Flow on Networks-Conservation Laws Models. AIMS Ser. Appl. Math. 2006, 1, doi:10.1.1.184.9484.

[13] Wu, N. Impact of traffic regulation on lane flow-distribution and capacity of motorways. In Proceeding of the Fifth International Conference of Transportation Professionals, Xian, China, 25-26 June 2005; pp. 24-26.

[14] Tian, Z.; Wu, N. Probability of capacity enhancement and disruption for freeway ramp controls: Analysis by gap-acceptance and queuing models. Transp. Res. Rec. J. Transp. Res. Board 2012, 2278, 1-12.

[15] Omnetpp. Available online: https://www.omnetpp.org/ (accessed on 10 March 2018).

[16] Veins. Available online: http://veins.car2x.org/ (accessed on 10 March 2018).

[17] Sumo. Available online: http://sumo.dlr.de/index.html (accessed on 10 March 2018).

[18] Arulkumar, N.; Raj, E.G.D.P. A simulation based study to implement Intelligent Transport Systems concepts in VANETs using AODV routing protocol in NS2. In Proceedings of the 2012 Fourth International Conference on Advanced Computing (ICoAC), Chennai, India, 13-15 December 2012; pp. 1-4. 